\title{
Perfil de ácidos graxos e conteúdo de ácido linoléico conjugado no leite de vacas alimentadas com a combinação de óleo de soja e fontes de carboidratos na dieta ${ }^{1}$
}

\author{
Eduardo da Costa Eifert ${ }^{2}$, Rogério de Paula Lana ${ }^{3}$, Dante Pazzanese Duarte Lanna ${ }^{4}$, Rafael \\ Monteiro Araújo Teixeira ${ }^{5}$, Pedro Braga Arcuri ${ }^{6}$, Maria Ignez Leão ${ }^{3}$, Marcus Vinícius Morais \\ de Oliveira ${ }^{7}$, Sebastião de Campos Valadares Filho ${ }^{3}$
}

\author{
${ }^{1}$ Parte da tese de Doutorado do primeiro autor, financiada pelo CNPq (processo 477530-01/0 NV). \\ 2 Pós-doc Nutrição de Ruminantes - ESALQ/USP. \\ ${ }^{3}$ Departamento de Zootecnia - UFV. \\ ${ }^{4}$ Departamento de Zootecnia-ESALQ/USP. \\ 5 Doutorando em Zootecnia - UFV. \\ ${ }^{6}$ Embrapa Gado de Leite. \\ 7 Universidade Estadual do Mato Grosso do Sul.
}

RESUMO - A gordura do leite de 12 vacas lactantes 7/8 Holandês-Gir foi utilizada para avaliar o efeito de diferentes dietas sobre o perfil dos ácidos graxos (AG) e o conteúdo de ácido linoléico conjugado (CLA) no leite. Os tratamentos consistiram de concentrados formulados com milho (MI) ou sua total substituição por farelo de trigo (FT) ou por polpa cítrica (PC), associados ou não a óleo de soja (0 e 2,25\%). A análise cromatográfica indicou que mais de $95 \%$ dos ácidos graxos (AG) foram recuperados. Não foi verificada interação significativa da presença de óleo e fonte de carboidratos em qualquer variável avaliada, à exceção das concentrações de trans-10 $\mathrm{C}_{18: 1}$ e da proporção de trans-10 $\mathrm{C}_{18: 1}$ e trans-11 $\mathrm{C}_{18: 1}$ nos totais trans- $\mathrm{C}_{18: 1}$ do leite. A fonte de carboidrato utilizada não influenciou o perfil de ácidos graxos do leite ou o teor de CLA. A inclusão de óleo de soja aumentou em $35 \%$ os AG insaturados do leite e reduziu em $14 \%$ os AG saturados, principalmente os de cadeia curta

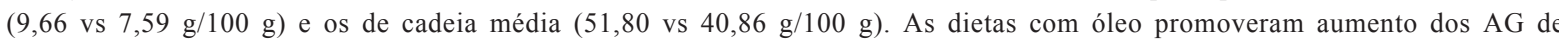
cadeia longa (32,17 vs 46,47 g/100 g), principalmente $\mathrm{C}_{18: 0}(9,44 \mathrm{vs} 13,63 \mathrm{~g} / 100 \mathrm{~g}), \mathrm{C}_{18: 1}(20,28 \mathrm{vs} 29,74 \mathrm{~g} / 100 \mathrm{~g})$ e $\mathrm{C}_{18: 2}$ $(2,16$ vs $2,92 \mathrm{~g} / 100 \mathrm{~g})$. A concentração de CLA cis-9 trans-11 $\mathrm{C}_{18: 2}$ foi aumentada em $230 \%(0,46 \mathrm{vs} 1,06 \mathrm{~g} / 100 \mathrm{~g})$ na presença de óleo. A concentração dos $\mathrm{AG}$ cis e trans- $\mathrm{C}_{18: 1}$ também foi aumentada; trans-11 $\mathrm{C}_{18: 1}$ foi o principal isômero trans, embora a concentração de trans-10 $\mathrm{C}_{18: 1}$ tenha se elevado de 0,28 para $1,11 \mathrm{~g} / 100 \mathrm{~g}$ na presença de óleo, sendo influenciada pela fonte de carboidrato. Os tratamentos MI, FT e PC resultaram em conteúdos deste isômero semelhantes na gordura do leite, mas, na presença de óleo, as fontes MI e FT proporcionaram valores muito superiores aos obtidos com PC, indicando diferentes rotas de biohidrogenação.

Palavras-chave: biohidrogenação, ácidos graxos do leite, lipídios, polpa cítrica, síntese de novo, subprodutos

\section{Milk fatty acid profile and milk conjugated linoleic acid content of dairy cows fed diets with different carbohydrate sources with or without soybean oil supplementation}

\begin{abstract}
Milk fat from 12 7/8 crossbred Holstein-Zebu lactating dairy cows was used to evaluate the effect of different diets on milk fatty acid (FA) profile and milk CLA content. Cows were fed concentrate containing corn (CR), wheat bran (WB), or dried citrus pulp (CP) supplemented or not with soybean oil. Chromatographic analysis showed a FA recovery greater than $95 \%$. With the exception of milk content of trans- $10 \mathrm{C}_{18: 1}$ and proportions of trans $-10 \mathrm{C}_{18: 1}$ and trans-11 $\mathrm{C}_{18: 1}$ on total milk trans- $\mathrm{C}_{18: 1}$, the interaction soybean oil $\mathrm{x}$ carbohydrate source was not significant for the remaining measured variables. Overall, carbohydrate source did not affect the milk FA profile or CLA content in this trial. Supplementation with soybean oil increased unsaturated FA by $35 \%$ and decreased saturated FA by $14 \%$, mainly short chain FA $(9.66 \mathrm{vs} .7 .59 \mathrm{~g} / 100 \mathrm{~g})$ and medium chain FA (51.80 vs. 40.86 g/100 g). Soybean oil increased long chain FA (32.17 vs. 46.47 g/100 g), mainly $C_{18: 0}$ (9.44 vs. $13.63 \mathrm{~g} / 100 \mathrm{~g}), \mathrm{C}_{18: 1}(20.28$ vs. $29.74 \mathrm{~g} / 100 \mathrm{~g})$ and $\mathrm{C}_{18: 2}(2.16 \mathrm{vs} .2 .92 \mathrm{~g} / 100 \mathrm{~g})$. Feeding soybean oil increased the concentration of cis-9 trans- $11 \mathrm{C}_{18.2}$ CLA by $230 \%$ (0.46 vs. $\left.1.06 \mathrm{~g} / 100 \mathrm{~g}\right)$. In addition, the content of cis and trans$\mathrm{C}_{18: 1}$ isomers was also increased. Although the trans-11 $\mathrm{C}_{18: 1}$ isomer was the most important, concentration of trans-10 $\mathrm{C}_{18: 1}$ increased from 0.28 to $1.11 \mathrm{~g} / 100 \mathrm{~g}$ in oil supplemented-diets being affected by the carbohydrate source. Milk from cows fed $\mathrm{CR}, \mathrm{WB}$, and $\mathrm{CP}$ showed similar content of trans-10 $\mathrm{C}_{18: 1}$. However, in diets with oil supplementation both $\mathrm{CR}$ and $\mathrm{WB}$ resulted in greater content of trans-10 $\mathrm{C}_{18: 1}$ than $\mathrm{CP}$ diet suggesting different biohydrogenation pathways.
\end{abstract}

Key Words: biohydrogenation, by-products, de novo synthesis, dried citrus pulp, lipids, milk fatty acids 


\section{Introdução}

É crescente o número de informações sobre a influência da gordura do leite e da carne de ruminantes sobre a saúde humana. Embora o leite de bovinos apresente alta concentração de ácidos graxos saturados, como os oriundos da síntese de novo, a literatura tem indicado que, no perfil da gordura do leite, há vários compostos benéficos à saúde humana.

Parodi (1999) enumerou uma série de compostos anticarcinogênicos presentes na gordura do leite, como o ácido linoléico conjugado (CLA), a esfingomielina, o ácido butírico, os éteres lipídicos, o $\beta$-caroteno e as vitaminas A e D. Especificamente em relação aos isômeros do CLA, efeitos anticarcinogênicos, antidiabéticos, de modulação do sistema imune, de partição da energia e de redução no desenvolvimento de aterosclerose têm sido reportados (Bauman et al., 2000), de modo que elevar o conteúdo de CLA tem-se tornado o foco de inúmeras pesquisas.

O CLA na carne e no leite de ruminantes tem origem a partir do CLA resultante da parcial biohidrogenação ruminal do ácido linoléico e da síntese endógena a partir da ação da enzima $\Delta^{9}$-desaturase sobre o trans-11 $\mathrm{C}_{18: 1}$ na glândula mamária, outro ácido graxo intermediário da biohidrogenação ruminal (Lawson et al., 2001).

De acordo com Bauman et al. (2000), cerca de $80 \%$ do CLA encontrado no leite é o isômero cis-9 trans- $11 \mathrm{C}_{18: 2}$. Estes autores têm estudado os fatores dietéticos que podem aumentar a concentração de CLA na gordura do leite, envolvendo a adição de lipídios na dieta, a alteração da relação volumoso:concentrado, a utilização de ionóforos e tampões e a utilização de pastagem, entre outros. Sem dúvida, as maiores respostas são observadas quando o ácido linoléico é adicionado à dieta.

Por vezes, tratamentos dietéticos que elevam o teor de CLA simultaneamente reduzem o teor de gordura do leite. Dhiman et al. (2000) observaram aumento de $237 \%$ no conteúdo de CLA em dietas com 2,5\% de óleo de soja, porém foi acompanhado por uma redução de $23 \%$ no teor de gordura do leite. Dietas contendo altas quantidades de grãos ou grãos contendo lipídios insaturados causam redução na gordura do leite, com aumentos nos totais de AG trans- $\mathrm{C}_{18: 1}$, diminuição nas concentrações de trans$11 \mathrm{C}_{18: 1}$ e concomitante aumento nas concentrações de trans-10 $\mathrm{C}_{18: 1}$ (Piperova et al., 2000).

Segundo Griinari et al. (1998), situações dietéticas em que o ambiente ruminal é alterado, por exemplo, baixa fibra efetiva e alta quantidade de grãos podem reduzir o $\mathrm{pH}$ ruminal e alterar a população microbiana causando desvio das rotas usuais de biohidrogenação ruminal, alterando a formação de cis-9 trans-11 $\mathrm{C}_{18: 2}$ e trans- $11 \mathrm{C}_{18: 1}$ para a formação de trans-10 cis-12 $\mathrm{C}_{18: 2}$ e trans-10 $\mathrm{C}_{18: 1}$ como principais intermediários da biohidrogenação do ácido linoléico. Trans-10 cis-12 $\mathrm{C}_{18: 2}$ e trans-10 $\mathrm{C}_{18: 1}$ estão envolvidos na inibição da atividade das enzimas acetil CoA carboxilase e ácido graxo sintetase na glândula mamária, responsável pela síntese de novo dos $\mathrm{AG}$ com até 16 carbonos (Piperova et al., 2000; Baumgard et al., 2002).

Os efeitos de diferentes fontes de carboidratos sobre o perfil de ácidos graxos e o conteúdo de CLA do leite têm sido pouco estudados. Solomon et al. (2000) não observaram interação grãos de soja extrusados $\times$ fonte de carboidrato sobre o conteúdo de CLA do leite quando o milho foi substituído por polpa cítrica no concentrado. A substituição do amido do milho por pectina da polpa cítrica tem alterado os padrões de fermentação ruminal, ocasionando maior formação de acetato e menor de propionato (Hall, 2001), mantendo o pH do rúmen mais alto (Leiva et al., 2000). Solomon et al. (2000) utilizaram grãos de soja extrusados como substrato à biohidrogenação para aumentar o conteúdo de CLA, pois esta fonte é considerada inerte sobre a população microbiana no rúmen. Butyrivibrio fibrisolvens é uma espécie bacteriana gram-negativa pertencente ao grupo A (responsável por converter o ácido linoléico em CLA) bastante versátil em relação à fermentação de diferentes substratos, podendo fermentar pectina ou amido, todavia, gera diferentes produtos finais da fermentação a partir destes substratos (Solomon et al., 2000).

A utilização de óleos insaturados tem efeitos sobre a população microbiana ao alterar a atividade da população celulolítica, influenciando a digestibilidade ruminal da fibra (Jenkins, 1993; Eifert et al., 2005). Assim, o óleo de soja pode apresentar efeitos diferentes aos grãos de soja extrusados, principalmente sobre a atividade das bactérias ruminais, como Butyrivibrio fibrisolvens, alterando a formação de CLA e o processo de biohidrogenação.

Este trabalho foi realizado com o objetivo de avaliar os efeitos da combinação de óleo de soja com diferentes fontes de carboidratos no concentrado sobre o perfil de ácidos graxos e o conteúdo de CLA da gordura do leite de vacas mestiças.

\section{Material e Métodos}

O experimento foi conduzido no período de dezembro de 2001 a março de 2002 no Setor de Pesquisa e Ensino em Gado de Leite do Departamento de Zootecnia da Universidade Federal de Viçosa.

Foram utilizadas 12 vacas multíparas lactantes (7/8 Holandês-Gir) com 86 dias em lactação, dispostas em quatro quadrados latinos $3 \times 3$. Os animais foram escolhidos aos 
pares e cada um foi alojado em um dos quadrados, de acordo com o histórico prévio de produção de leite, o estádio de lactação e o número de parições.

Os tratamentos consistiram da substituição total do milho no concentrado (MI) por farelo de trigo (FT) ou polpa cítrica (PC), associados ou não ao óleo de soja ( 0 e 2,25\% de óleo de soja). O delineamento foi um ensaio fatorial $3 \times 2$, com dois níveis de óleo e três fontes de carboidratos.

Cada período experimental teve duração de 17 dias, dez para adaptação e sete para coleta das amostras e dos resultados. Os animais foram alojados em baias individuais, tipo tie-stall, sendo alimentados e ordenhados duas vezes ao dia, com controle diário de oferta e sobras dos alimentos. As dietas continham 55,2\% de silagem de milho e $44,8 \%$ de concentrado e, em média, $15,5 \%$ de PB e $39,3 \%$ de FDN (Tabela 1). As dietas foram formuladas a partir das estimativas do NRC (2001) para atender às necessidades de vacas com produção de $20 \mathrm{~kg} /$ dia e leite com 3,5\% de gordura.

A produção de leite foi medida diariamente e amostras do leite de dois dias consecutivos de ordenha foram retiradas para avaliação da composição do leite, realizada no Laboratório de Qualidade do Leite da Embrapa Gado de Leite, por meio da análise do infravermelho em um aparelho Bentley 2000 (resultados apresentados em um artigo seqüencial, Eifert et al., 2006). Uma alíquota de $2 \%$ da produção diária foi retirada e congelada para a posterior análise do perfil de $\mathrm{AG}$ do leite. A extração da gordura foi realizada com base no método descrito por Nourooz-Zadeh \& Appelqvist (1988): $17 \mathrm{~mL}$ de leite (em duplicata) foram transferidos para um funil de separação, acrescidos de $30 \mathrm{~mL}$ de isopropanol e, após agitação, foram adicionados de $22,5 \mathrm{~mL}$ de hexano e agitados por mais 3 minutos. A mistura foi centrifugada a $2.520 \mathrm{~g}$ por 5 minutos a $5^{\circ} \mathrm{C}$ e a camada superior foi transferida para outro funil de separação. A camada inferior foi extraída mais duas vezes com $22,5 \mathrm{~mL}$ de hexano e esses extratos foram adicionados ao primeiro. A água foi retirada dos extratos com a adição de $15 \mathrm{~mL}_{\text {de }} \mathrm{Na}_{2} \mathrm{SO}_{4} 0,47 \mathrm{M}$. A camada de hexano foi coletada em frasco e evaporada a $50^{\circ} \mathrm{C}$ em evaporador rotativo com fluxo de nitrogênio contínuo.

A transesterificação dos ácidos graxos foi realizada conforme descrito por Eifert et al. (2006a), segundo o protocolo de Christie (1982), com as adaptações de Chouinard et al. (1998), utilizando-se metóxido de sódio como agente de metilação. O perfil de ácidos graxos foi determinado por cromatografia gasosa utilizando-se coluna capilar de $100 \mathrm{~m}$ de sílica fundida (SP-2560) e detector de ionização de chama (FID). A corrida teve duração de 70 minutos, utilizando-se nitrogênio como gás de arraste a um fluxo de $40 \mathrm{~mL} /$ minuto e gradiente de temperatura. Uma manteiga padrão (CRM 164; Commission of the European Communities, Community

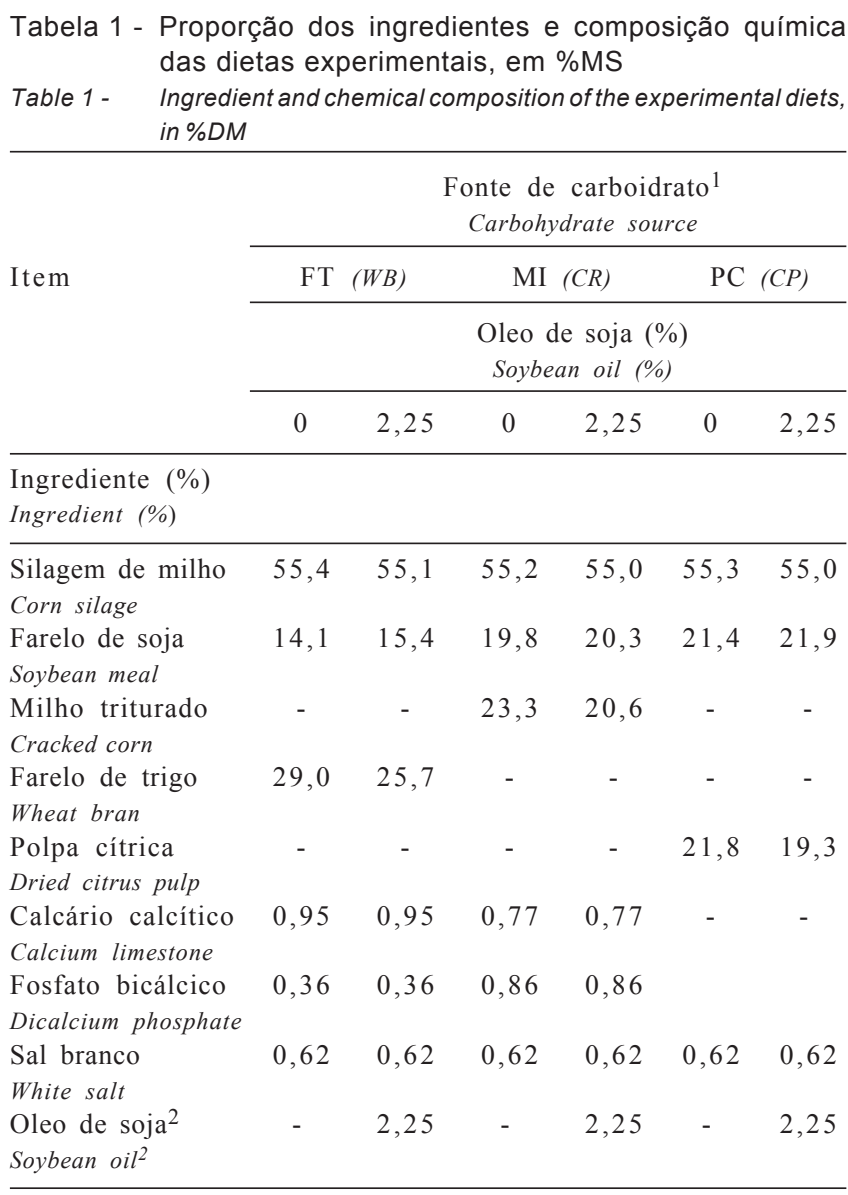

Composição química (\%)

Chemical composition (\%)

\begin{tabular}{lrrrrrr}
\hline MO (OM) & 93,4 & 93,5 & 93,1 & 93,2 & 94,0 & 94,1 \\
FDN (NDF) & 45,4 & 44,0 & 35,3 & 35,0 & 38,2 & 37,6 \\
FDA (ADF) & 26,0 & 25,2 & 20,3 & 20,3 & 22,6 & 22,2 \\
PB (CP) & 15,7 & 15,9 & 15,7 & 15,9 & 15,6 & 15,8 \\
EE & 2,8 & 5,0 & 2,8 & 4,9 & 2,4 & 4,6 \\
\hline
\end{tabular}

Ácidos graxos, g/100 g

Fatty acids, $\mathrm{g} / 100 \mathrm{~g}$

\begin{tabular}{lrrrrrr}
\hline $\mathrm{C}_{12: 0}$ & 2,30 & 1,35 & 2,31 & 1,35 & 3,05 & 1,63 \\
$\mathrm{C}_{14: 0}$ & 0,80 & 0,46 & 0,24 & 0,18 & 1,57 & 0,80 \\
$\mathrm{C}_{16: 0}$ & 16,62 & 13,65 & 13,21 & 11,93 & 19,20 & 14,62 \\
$\mathrm{C}_{18: 0}$ & 3,56 & 3,65 & 2,58 & 3,15 & 4,06 & 3,89 \\
$\mathrm{C}_{18: 1}$ & 20,01 & 21,24 & 20,80 & 21,64 & 20,27 & 21,45 \\
$\mathrm{C}_{18: 2}$ & 49,86 & 50,39 & 52,94 & 51,94 & 42,64 & 47,06 \\
$\mathrm{C}_{18: 3}$ & 1,65 & 4,06 & 2,39 & 4,43 & 2,94 & 4,84 \\
Outros & 5,20 & 5,21 & 5,53 & 5,38 & 6,27 & 5,70 \\
Others & & & & & &
\end{tabular}

${ }^{1} \mathrm{FT}=$ farelo de trigo; $\mathrm{Ml}=$ milho; $\mathrm{PC}=$ polpa cítrica .

2 Óleo de soja foi misturado ao concentrado.

${ }^{1} \mathrm{WB}=$ wheat bran; $C R=$ corn; $C P=$ citrus pulp.

2 Soybean oil was mixed on the concentrate.

Bureau of Reference, Brussels, Belgium) foi utilizada na determinação dos fatores de recuperação para os ácidos graxos, cujas concentrações foram expressas em g/100 g de ácidos graxos totais. As análises cromatográficas e o processo de transesterificação dos ácidos graxos do leite foram realizados no Laboratório de Nutrição e Crescimento Animal daESALQ-USP. 
As vacas foram dispostas em dois quadrados latinos 3 x 3 com óleo e em outros dois quadrados latinos sem óleo. Para avaliar o efeito de óleo, optou-se por uma modificação do desenho split-plot, utilizando "vaca dentro de óleo" como erro "b". Este tipo de análise foi utilizado por outros pesquisadores (Drackley et al., 2001; Elliott et al., 1995). As demais fontes de variação adicionadas ao modelo foram a fonte de carboidratos, o óleo e a interação fonte de carboidratos $\times$ óleo, vaca e período. Para minimizar a variação do animal entre os quadrados, todos os animais foram pareados e cada vaca do par foi inserida em um quadrado com óleo e a outra vaca, no quadrado sem óleo. Para a seleção dos pares de vacas, todos os animais permaneceram sob o mesmo regime alimentar dez dias antes do início do experimento, quando a produção de leite foi medida. Assim, os critérios para escolha dos animais foram a produção de leite atual, a produção na lactação anterior, o peso, o estádio de lactação e o número de parições. Os dados foram submetidos à análise estatística com o auxílio do programa estatístico SAS (1999), adotando-se o nível de significância de 5\% na discussão dos resultados.

\section{Resultados e Discussão}

A análise cromatográfica indicou que mais de $95 \%$ dos ácidos graxos foram recuperados e seus valores estão expressos em $\mathrm{g} / 100 \mathrm{~g}$ do total recuperado. Os diferentes carboidratos não influenciaram o perfil da gordura do leite (Tabela 2) e as maiores alterações foram verificadas quando o óleo foi adicionado às dietas. A inclusão de óleo de soja reduziu a concentração dos ácidos graxos de cadeia curta (AGCC) e de cadeia média (AGCM) em 22,0 e 23,2\%, respectivamente. Individualmente, o butirato $\left(\mathrm{C}_{4: 0}\right)$ foi o único $\mathrm{AG}$ que não inibido pelo óleo de soja, pois este ácido graxo pode ser secretado após o $\beta$-hidroxibutirato sofrer $\beta$-redução, não dependendo da participação de malonil-CoA ou da ação da acetil CoA carboxilase (Fox \& McSweeney, 1998). A consistência nas proporções de butirato é interessante, particularmente pelos efeitos positivos do ácido butírico sobre a saúde humana (Parodi, 1999; Chilliard et al., 2000).

Considerando que o óleo de soja utilizado apresentou $51 \%$ de ácido linoléico, $6,8 \%$ de linolênico e $22,8 \%$ de oléico, as alterações nos AGCC e AGCM são coerentes com as afirmações de Grummer (1991) de que AG de cadeia longa inibem a incorporação de $\mathrm{AG}$ de cadeias média e curta na gordura do leite, que envolve a enzima acetil-CoA carboxilase, embora os mecanismos não estejam totalmente elucidados. De acordo com Palmquist et al. (1993), ácidos graxos de cadeia longa na dieta ou oriundos da mobilização corpórea resultante do balanço energético negativo (BEN) inibem a síntese de novo na glândula mamária. Este processo é dinâmico e, à medida que o animal sai do BEN, há um concomitante incremento de ácidos graxos de 6 a 12 carbonos, chegando a um patamar superior a partir da $8^{\mathrm{a}}$ semana de lactação (Eifert et al., 2006a).

A concentração dos ácidos graxos de cadeia ímpar não foi influenciada pela fonte de carboidratos, mas a inclusão de óleo de soja reduziu sua concentração em 16,1\%, principalmente $\mathrm{C}_{13: 0}$ e $\mathrm{C}_{15: 0}$ (Tabela 2). A principal fonte destes ácidos graxos são as bactérias ruminais que sintetizam AG a partir dos ácidos graxos voláteis de cadeia ímpar, como o propionato e o valerato, e são incorporada à sua estrutura de membrana. Pouco se sabe a respeito de como bactérias regulam sua composição lipídica, entretanto, sabe-se que bactérias podem incorporar diretamente os ácidos graxos da dieta. Possivelmente, este processo tenha ocorrido à medida que a síntese de ácidos graxos monoinsaturados de origem microbiana, por exemplo $\mathrm{C}_{10: 1}$, também foi inibida na presença de óleo, o que está de acordo com os dados reportados por Mansbridge \& Blake (1997), Solomon et al. (2000) e Eifert et al. (2006a).

Concomitantemente à redução dos AG de cadeias curta e média, os ácidos graxos de cadeia longa aumentaram em 29,6\% na presença de óleo na dieta. A inclusão do óleo de soja também diminuiu a proporção dos ácidos graxos saturados em 13,2\% e aumentou a participação dos ácidos graxos insaturados, monoinsaturados (MUFA) e poliinsaturados (PUFA) em 33,8;33,0 e 41,8\%, respectivamente. Ácidos graxos poliinsaturados não são sintetizados pelos tecidos dos ruminantes e, portanto, sua concentração no leite é dependente da quantidade destes ácidos graxos que chegam ao duodeno (Chilliard et al., 2000). Também correspondendo ao perfil de ácidos graxos do óleo de soja, observaram-se aumentos na concentração de $\mathrm{C}_{18: 2}$ e $\mathrm{C}_{18: 1}$ (Tabela 2), embora a suplementação com lipídios insaturados não tenha promovido alteração na concentração de $\mathrm{C}_{18: 3}$.

Óleos adicionados às dietas de ruminantes são extensivamente biohidrogenados no retículo-rúmen. A biohidrogenação de óleo de girassol foi de $80 \%$ em vacas lactantes (Kalscheur et al., 1997), enquanto a do ácido linoléico foi de $75 \%$ quando até $8 \%$ de óleo de soja foi adicionado na dieta de vacas não-lactantes (Bateman \& Jenkins, 1998). Neste trabalho, apesar do aumento na concentração de esteárico $\left(\mathrm{C}_{18: 0}\right.$, Tabela 2$)$, o aumento verificado na concentração dos ácidos graxos trans- $\mathrm{C}_{18-1}$ indica que a biohidrogenação não foi completa no retículorúmen (Tabela 3), embora seja comum que a extensão da biohidrogenação aumente com a elevação do grau de 
Tabela 2 - Perfil dos ácidos graxos do leite de vacas alimentadas com diferentes fontes de carboidratos e óleo de soja Table 2 - Milk fatty acid profile of cow EPMs fed different carbohydrate sources and soybean oil

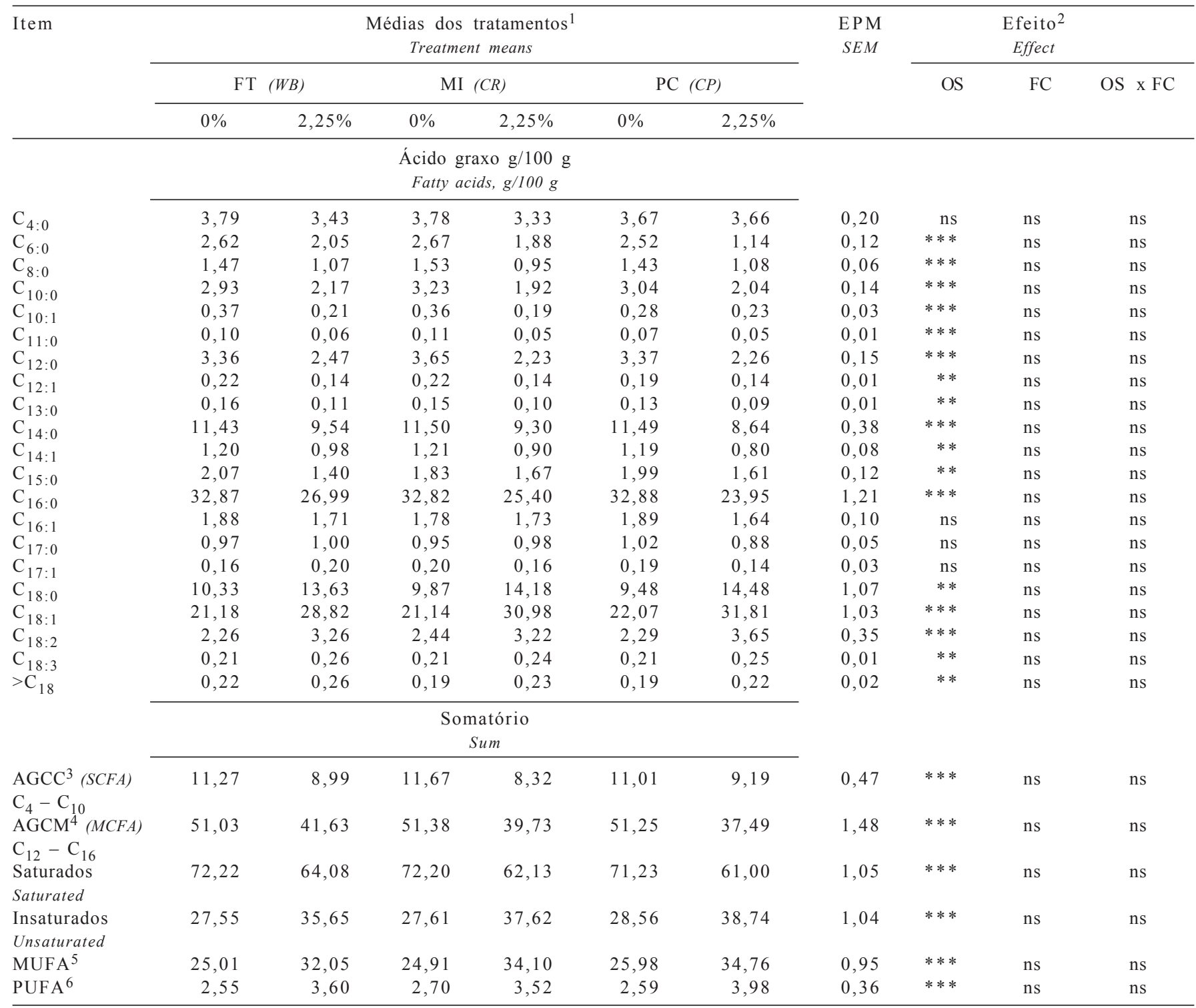

${ }_{1}^{1} \mathrm{MI}=$ milho; $\mathrm{FT}=$ farelo de trigo; $\mathrm{PC}=$ polpa cítrica.

2 OS = efeito do óleo de soja; FC - efeito da fonte de carboidrato; OS $\times F C$ - interação; ${ }^{*}(P<0,05) ;{ }^{* *}(P<0,01) ;{ }^{* * *}(P<0,001)$.

${ }^{3} \sum \mathrm{C}_{4}-\mathrm{C}_{10} ;{ }^{4} \sum \mathrm{C}_{12}-\mathrm{C}_{16}$.

5 Ácidos graxos monoinsaturados.

6 Ácidos graxos poliinsaturados.

${ }^{1} C R=$ corn; $W B=$ wheat bran; $C P=$ citrus pulp.

${ }^{2}$ OS = soybean oil effect; $F C-$ carbohydrate source effect; OS $\times F C-$ interaction; ${ }^{*}(P<0.05) ;{ }^{* *}(P<0.01) ;{ }^{* * *}(P<0.001)$.

${ }^{5}$ Monounsaturated fatty acids.

6 Polyunsaturated fatty acids.

insaturação da dieta (Kalscheur et al., 1997), justificando os aumentos no ácido esteárico $\left(\mathrm{C}_{18: 0}\right)$ nas dietas com óleo de soja.

A concentração do ácido linoléico (cis-9 cis-12 $\mathrm{C}_{18: 2}$ ) não diferiu entre as fontes de carboidratos, mas aumentou na presença de óleo (Tabela 3 ). O pequeno acréscimo observado $(1,85$ vs $2,21 \mathrm{~g} / 100 \mathrm{~g})$ em relação à quantidade de linoléico adicionado nas dietas indica intensa biohidrogenação deste AG e, possivelmente, a preferência de utilização por outros órgãos e tecidos, como o reprodutivo (Chilliard et al., 2000). Esta mesma resposta foi observada em outros estudos nos quais foram utilizadas fontes ricas em linoléico na dieta (Chilliard et al., 2000; Eifert et al., 2006a). As dietas com óleo não elevaram a concentração de trans-11 cis-15 $\mathrm{C}_{18: 2}$, possivelmente por este AG ser preferencialmente biohidrogenado em relação aos demais insaturados e porque, como é intermediário da biohidrogenação do ácido linolênico, sua pequena participação corresponde à proporção de $\mathrm{C}_{18: 3}$ na gordura do leite. 
Tabela 3 - Perfil dos ácidos graxos cis e trans octadecenóicos do leite de vacas alimentadas com diferentes fontes de carboidratos e óleo de soja, $\mathrm{g} / 100 \mathrm{~g}$

Table 3 - $\quad$ Content of cis and trans octadecenoic acids on milk fat of cows fed diets with different carbohydrate sources and soybean oil, $\mathrm{g} / 100 \mathrm{~g}$

\begin{tabular}{|c|c|c|c|c|c|c|c|c|c|c|}
\hline \multirow[t]{2}{*}{ Item } & \multicolumn{6}{|c|}{$\begin{array}{c}\text { Médias dos tratamentos } \\
\text { Treatment means }\end{array}$} & \multirow[t]{2}{*}{$\begin{array}{l}\text { Е P M } \\
S E M\end{array}$} & \multicolumn{3}{|c|}{$\begin{array}{c}\text { Efeito }^{2} \\
\text { Effect }\end{array}$} \\
\hline & \multicolumn{2}{|c|}{$\mathrm{FT}(W B)$} & \multicolumn{2}{|c|}{$\mathrm{MI}(C R)$} & \multicolumn{2}{|c|}{$\mathrm{PC}(C P)$} & & OS & $\mathrm{FC}$ & $\mathrm{OS} \times \mathrm{FC}$ \\
\hline & \multicolumn{6}{|c|}{ Isômeros $\mathrm{C}_{18: 1}$ (Isomers $C_{18: 1}$ ) } & & & & \\
\hline Trans- $\mathrm{C}_{18: 1}$ & 1,59 & 4,20 & 1,68 & 4,22 & 2,21 & 4,15 & 0,59 & $* * *$ & ns & $\mathrm{ns}$ \\
\hline trans -10 & 0,21 & 1,42 & 0,23 & 1,29 & 0,40 & 0,59 & 0,21 & $* * *$ & ns & 0,0565 \\
\hline $\operatorname{trans}-11$ & 0,72 & 1,49 & 0,73 & 1,51 & 0,99 & 2,27 & 0,42 & * & ns & $\mathrm{ns}$ \\
\hline $\operatorname{trans}-12$ & 0,21 & 0,41 & 0,24 & 0,44 & 0,27 & 0,43 & 0,06 & $* *$ & ns & $\mathrm{ns}$ \\
\hline $\operatorname{trans}-16$ & 0,14 & 0,25 & 0,14 & 0,27 & 0,16 & 0,26 & 0,03 & $* * *$ & ns & ns \\
\hline Cis- $\mathrm{C}_{18: 1}$ & 19,59 & 24,62 & 19,46 & 26,76 & 19,89 & 27,67 & 0,95 & $* * *$ & ns & $\mathrm{ns}$ \\
\hline cis -9 & 18,79 & 23,43 & 18,07 & 25,15 & 18,67 & 26,35 & 0,88 & $* * *$ & ns & $\mathrm{ns}$ \\
\hline cis -15 & \multicolumn{6}{|c|}{ Isômeros $\mathrm{C}_{18: 2} \quad$ (Isomers $C_{18: 2}$ ) } & & & & \\
\hline Cis-9 cis-12 & 1,80 & 2,30 & 2,01 & 2,21 & 1,74 & 2,12 & 0,16 & $*$ & ns & ns \\
\hline Cis -9 trans -11 & 0,46 & 0,92 & 0,44 & 0,97 & 0,58 & 1,50 & 0,23 & * & ns & ns \\
\hline Trans -10 cis-12 & 0,00 & 0,016 & 0,00 & 0,017 & 0,00 & 0,003 & 0,00 & $*$ & ns & $\mathrm{ns}$ \\
\hline Trans-11 cis-15 & 0,00 & 0,03 & 0,00 & 0,02 & 0,00 & 0,03 & 0,02 & $\mathrm{~ns}$ & ns & ns \\
\hline
\end{tabular}

${ }_{1} \mathrm{MI}=$ milho; $\mathrm{FT}=$ farelo de trigo; $\mathrm{PC}=$ polpa cítrica.

2 OS = efeito do óleo de soja; FC - efeito da fonte de carboidrato; OS $\times F C$ - interação; * $(P<0,05)$; ** $(P<0,01)$; $* * *(P<0,001)$.

${ }^{1} C R=$ corn; $W B=$ wheat bran; $C P=$ citrus pulp.

2 OS = soybean oil effect; $F C$ - carbohydrate source effect; OS $\times F C-$ interaction; * $(P<0.05) ;{ }^{* *}(P<0.01) ; * * *(P<0.001)$

Nos últimos anos, têm-se discutido sobre o papel dos isômeros trans gerados no rúmen durante a biohidrogenação; o acúmulo destes isômeros pode ser função da menor toxicidade da configuração trans às membranas celulares quando comparada à configuração cis (Bessa et al., 2000). A rota da biohidrogenação do ácido linoléico, como tradicionalmente descrita, envolve a formação de um dieno conjugado e a posterior formação de um isômero trans até a completa saturação ao esteárico $\left(\mathrm{C}_{18: 0}\right)$. Embora a formação de CLA cis-9 trans-11 $\mathrm{C}_{18: 2}$ e de trans-11 $\mathrm{C}_{18: 1}$ esteja envolvida na principal rota de biohidrogenação, em situações dietéticas específicas, há o aparecimento de outros dienos e AG trans correspondentes (Chilliard et al., 2000) conforme o aumento das concentrações dos diferentes trans- $\mathrm{C}_{18: 1}$ no leite (Tabela 3).

A concentração dos isômeros trans- $\mathrm{C}_{18: 1}$ foi aumentada em $229 \%$ e a de cis- $\mathrm{C}_{18: 1}$ em 33,8\% quando o óleo de soja foi adicionado às dietas (Tabela 3). O ácido oléico, cis-9 $\mathrm{C}_{18: 1}$, foi o principal isômero cis, resultante tanto da dieta como da ação da $\Delta^{9}$-desaturase sobre o ácido esteárico (Jenkins, 1993). O ácido vacênico, trans-11 $\mathrm{C}_{18: 1}$, foi o principal isômero trans. Se, por um lado, somente as concentrações dos ácidos oléico e cis-12 $\mathrm{C}_{18: 1}$ foram aumentadas nos ácidos cis, todos os isômeros trans $-\mathrm{C}_{18: 1}$ recuperados foram aumentados na presença de óleo de soja.

As fontes de carboidratos não apresentaram diferenças estatísticas $(\mathrm{P}>0,10)$ em relação às concentrações de trans-11 $\mathrm{C}_{18: 1}$ no leite, no entanto, a dieta com polpa cítrica apresentou valores $53 \%$ maiores que as demais fontes de carboidratos. Verificou-se interação $(P=0,0565)$ fonte de carboidratos $\times$ óleo de soja sobre a concentração de trans-10 $\mathrm{C}_{18: 1}$ (Figura 1A), de modo que, na ausência de óleo, a concentração de trans-10 $\mathrm{C}_{18: 1}$ foi semelhante entre as fontes de carboidratos, em média, de $0,28 \mathrm{~g} / 100 \mathrm{~g}$. Em MI e FT, a concentração de trans-10 $\mathrm{C}_{18: 1}$ foi aumentada na presença de óleo, no entanto, este $A G$ não foi alterado quando o óleo foi adicionado na dieta PC. Quando as concentrações de trans-10 e trans- $11 \mathrm{C}_{18: 1}$ foram relacionadas aos ácidos graxos trans- $\mathrm{C}_{18: 1}$ no leite (Figuras 1B e 1C), estes AG mostraram interação significativa óleo $\times$ fonte de carboidratos $(\mathrm{P}=0,0525 \mathrm{e}$ $\mathrm{P}=0,0970$, respectivamente).

De acordo com as Figuras 1B e 1C, a manutenção da proporção de trans-10 e o aumento na proporção de trans-11 $\mathrm{C}_{18: 1}$ em relação aos totais trans $-\mathrm{C}_{18: 1}$, quando o óleo de soja foi adicionado na dieta PC reforça 
A

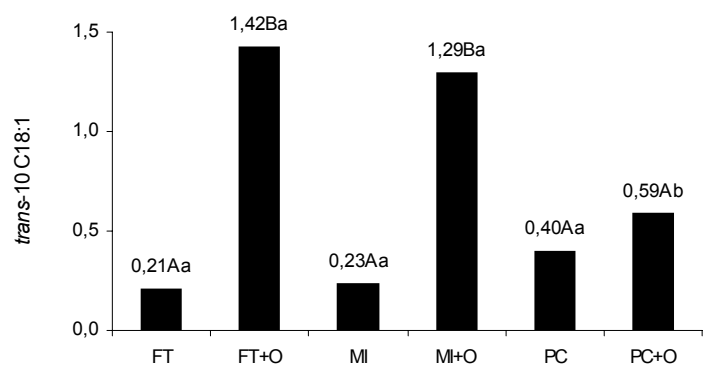

B

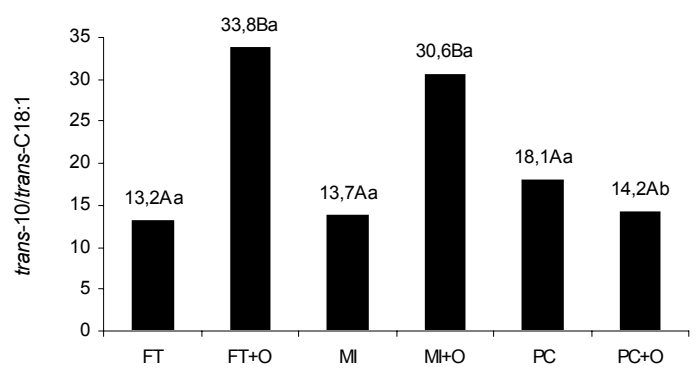

C

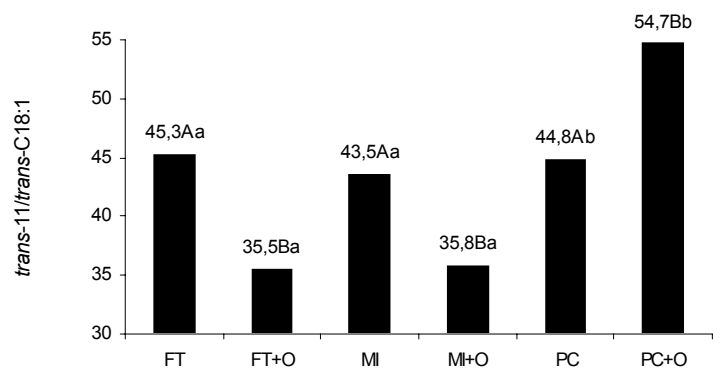

Figura 1 - Concentração de trans-10 $\mathrm{C}_{18: 1}$ na gordura do leite (1A) e proporção de trans-10 (1B) e trans-11 $\mathrm{C}_{18: 1}$ (1C) nos totais de trans- $C_{18: 1}$ no leite. A e B indicam diferença entre as fontes de carboidratos; $a$, b indicam diferenças dentro das dietas com e sem óleo (Tukey, $\mathrm{P}<0,05)$. FT, MI, PC - fontes de carboidratos; $\mathrm{FT}+\mathrm{O}$, $\mathrm{MI}+\mathrm{O}, \mathrm{PC}+\mathrm{O}-$ fontes de carboidratos com óleo de soja.

Figure 1 - Concentration of trans-10 $C_{18: 1}$ in milk fat (1A) and proportion of trans-10 (1B) and trans-11 $C_{18: 1}(1 C)$ on total trans- $C_{18: 1}$ in milk. " $A$ ", " $B$ ", shows difference between carbohydrate sources; "a", " $b$ ", shows difference with or without oil supplementation (Tukey, $P<0.05)$. WB, $C R, C P$ - carbohydrate sources; $W B+O$, $\mathrm{CR}+\mathrm{O}, \mathrm{CP}+\mathrm{O}-$ carbohydrate source with soybean oil.

que a polpa cítrica permitiu um ambiente ruminal mais apropriado para a rota da biohidrogenação envolvendo o CLA cis-9 trans-11 $\mathrm{C}_{18: 2}$ e trans-11 $\mathrm{C}_{18: 1}$. Neste sentido, Bradford \& Allen (2004), ao compararem a substituição do milho triturado pelo milho úmido no concentrado, sugeriram que a velocidade de fermentação dos carboidratos pode alterar a concentração de trans-10 $\mathrm{C}_{18: 1}$ no leite. Estes autores observaram que o milho úmido aumentou trans-10 $\mathrm{C}_{18: 1}$ no leite de vacas de $18-30 \mathrm{~kg}$ de produção (como neste trabalho), porém, não aumentou nas de alta produção, sugerindo que a maior velocidade de passagem da digesta, pelo maior consumo, pode ter mantido o ambiente ruminal propício à rota de biohidrogenação que envolve CLA cis-9 trans-11 $\mathrm{C}_{18: 2}$ e trans-11 $\mathrm{C}_{18: 1}$, independentemente da taxa de fermentação do milho utilizado.

Associados às taxas de fermentação, outros fatores envolvidos na rota normal de biohidrogenação são a manutenção do ambiente ruminal e o fornecimento de substrato para o crescimento de microrganismos específicos. Neste sentido, sabe-se que a polpa cítrica tem apresentado padrões de fermentação que mantêm o pH ruminal mais alto que grãos ricos em amido (Leiva et al., 2000), o que, possivelmente, tenha contribuído para o crescimento das bactérias celulolíticas, principais envolvidas na isomeração do ácido linoléico para CLA cis-9 trans-11 $\mathrm{C}_{18: 2}$ (Harfoot \& Hazlewood, 1997). O ambiente ruminal com o $\mathrm{pH}$ mais alto, associado ao fornecimento de pectina como substrato, pode ter favorecido o crescimento de Butyrivibrio fibrisolvens na dieta $\mathrm{PC}$, também relacionada à formação de CLA cis-9 trans-11 $\mathrm{C}_{18: 2}$ e à redução para trans-11 $\mathrm{C}_{18: 1}$ (Solomon et al., 2000). Por outro lado, Kim et al. (2002) demostraram que Megasphaera elsdenii, que utiliza lactato para seu crescimento, produz CLA trans-10 cis-12 $\mathrm{C}_{18: 2}$ como principal dieno na biohidrogenação do ácido linoléico. Assim, esta espécie microbiana poderia ter seu crescimento estimulado nas dietas FT e MI, originando os níveis de trans-10 $\mathrm{C}_{18: 1}$ observados.

Não foi detectado o CLA trans-10 cis-12 $\mathrm{C}_{18: 2}$ no leite dos animais que receberam as dietas sem óleo (Tabela 3 ) e o nível detectado nas dietas com óleo foi baixo $(0,012 \mathrm{~g} / 100 \mathrm{~g})$ quando comparado aos valores reportados na literatura (Bauman \& Griinari, 2001), o que indica alta biohidrogenação sobre este AG. Embora de pouca magnitude, a dieta $\mathrm{PC}$ resultou em menor concentração de CLA trans-10 cis-12 $\mathrm{C}_{18: 2}$ na gordura do leite que as dietas FT e MI, coincidindo com as menores concentrações de trans-10 $\mathrm{C}_{18: 1}$ na dieta PC.

Embora não se tenha um produto comercial puro trans-10 $\mathrm{C}_{18: 1}$, este $\mathrm{AG}$ e o CLA trans-10 cis-12 $\mathrm{C}_{18: 2}$ estão envolvidos na redução do teor de gordura do leite (Bauman \& Griinari, 2001). Neste trabalho, o teor de gordura do leite não foi influenciado pela fonte de carboidrato, com média de 3,24\%, mas foi reduzido de 3,34 para 3,13\% quando o óleo de soja foi adicionado nas dietas (Eifert et al., 2006). O AG trans-10 $\mathrm{C}_{18: 1}$ apresentou melhor correlação que o CLA trans- 10 cis- $12 \mathrm{C}_{18: 2}$ com o teor de gordura $(\mathrm{r}=-0,4885$ $\mathrm{P}=0,0025$ vs $\mathrm{r}=-0,3663 \mathrm{P}=0,0280)$ e os ácidos graxos de cadeias curta $(\mathrm{r}=-0,5581 \mathrm{P}=0,0002 \mathrm{vsr}=-0,4477 \mathrm{P}=0,0062)$ emédia $(\mathrm{r}=-0,5178 \mathrm{P}=0,0012$ vsr $=-0,3361 \mathrm{P}=0,0451)$. Como anteriormente apresentado na Tabela 3 , a redução no teor de gordura pode ser atribuída às diminuições dos ácidos graxos resultantes da síntese de novo, os AG de cadeias média $(23,2 \%)$ e curta $(22,0 \%)$. 
De forma similar à ocorrência de trans- $11 \mathrm{C}_{18: 1}$, as fontes de carboidratos não diferiram entre si $(\mathrm{P}>0,10)$ quanto ao conteúdo de CLA cis-9 trans-11 $\mathrm{C}_{18: 2}$ e a inclusão de óleo de soja na dieta proporcionou aumentos de $230 \%$ no conteúdo deste CLA (Tabela 3). Embora sem interação significativa, $\mathrm{PC}$ apresentou maior valor deste CLA que FT e MI tanto nas dietas sem $(0,58$ vs 0,44 e $0,46 \mathrm{~g} / 100 \mathrm{~g})$ quanto naquelas com óleo de soja ( $1,50 \mathrm{vs} 0,92$ e $0,97 \mathrm{~g} / 100 \mathrm{~g}$ ). Os valores encontrados corroboram as afirmações de Bauman et al. (2000) e Chilliard et al. (2000) de que fontes ricas em ácido linoléico, como o óleo de soja, são muito efetivas em elevar as concentrações deste CLA. Os valores médios observados de CLA cis-9 trans-11 $\mathrm{C}_{18: 2}$ foram de 0,46 e 1,06 g/100 g para a ausência e presença de óleo, respectivamente. Estes valores confirmam os encontrados por Dhiman et al. (2000), que verificaram que a inclusão de 2,5\% de óleo de soja permitiu aumento de $237 \%$

A

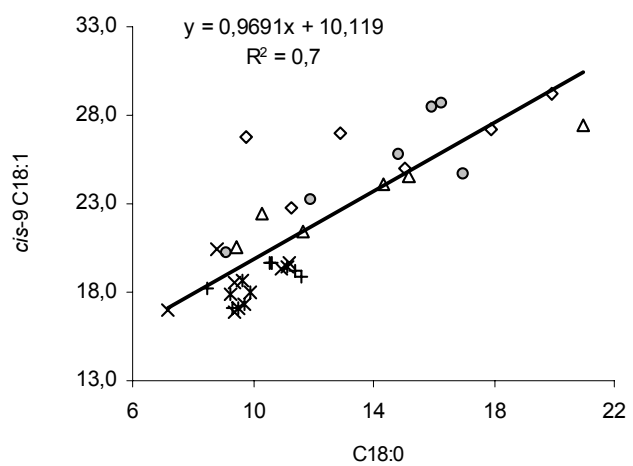

B
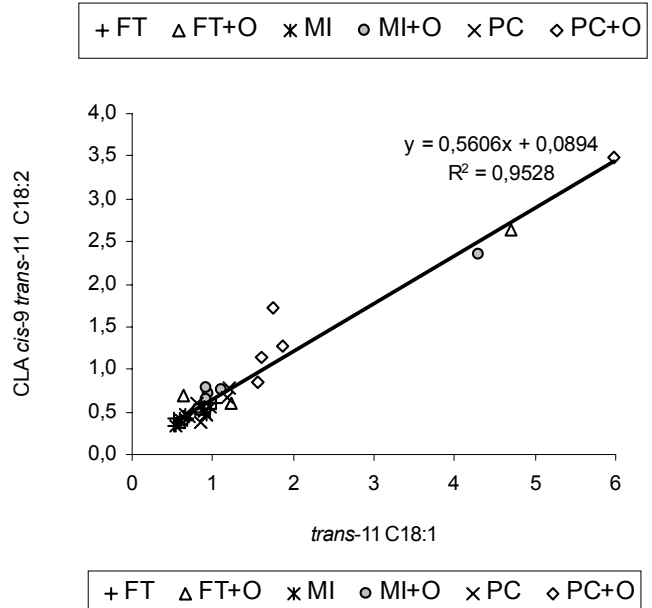

Figura 2 - Relação da concentração dos ácidos oléico (cis-9 $\left.\mathrm{C}_{18: 1}\right)$ e esteárico $\left(\mathrm{C}_{18: 0}\right)$ na gordura do leite (2A) e a concentração de CLA cis- 9 trans-11 $\mathrm{C}_{18.2}$ e trans-11 $\mathrm{C}_{18: 1}(2 \mathrm{~B})$. $\mathrm{FT}, \mathrm{MI}, \mathrm{PC}$ - fontes de carboidratos; $\mathrm{FT}+\mathrm{O}, \mathrm{MI}+\mathrm{O}, \mathrm{PC}+\mathrm{O}$ - fontes de carboidratos com óleo de soja.

Figure 2 - Relationship between milk fat concentration of oleic acid (cis-9 $\left.C_{18: 1}\right)$ and stearic acid $\left(C_{18: 0}\right)(2 A)$, and the concentration of cis- 9 trans-11 $C_{18: 2} C L A$ and trans- $11 C_{18: 1}$ (2B). $W B, C R, C P$ - carbohydrate sources; $W B+O, C R+O$, $C P+O$ - carbohydrate source with soybean oil. do CLA. Chilliard et al. (2000), em revisão de literatura, observaram que valores de CLA entre 0,8 e 1,6\% da gordura do leite são esperados para esta dose de óleo de soja.

Conforme demonstrado na Figura 2, a atividade da $\Delta{ }^{9}$-desaturase não foi alterada pelos tratamentos. Na Figura 2A consta a linearidade da relação do ácido esteárico e o cis-9 $\mathrm{C}_{18: 1}$, embora as dietas com óleo indiquem maior variação da distribuição destes $A G$ que as dietas sem óleo, o que reflete a dinâmica da biohidrogenação e de seus intermediários. A similaridade das respostas do CLA cis- 9 trans- $11 \mathrm{C}_{18: 2}$ com as observadas para trans-11 $\mathrm{C}_{18: 1}$ indicam a forte influência da atividade da $\Delta^{9}$-desaturase na secreção deste CLA. O CLA cis-9 trans-11 $\mathrm{C}_{18: 2}$ apresentou valores muito próximos nas dietas sem óleo, porém, houve grande dispersão dos resultados nas dietas com óleo, explicada pela maior formação de trans-11 $\mathrm{C}_{18: 1}$ no rúmen e pela alta atividade de desaturação. Outros autores têm reportado grande variação da desaturase entre animais, como Solomon et al. (2000), que observaram três vezes a variação da atividade desta enzima entre os animais testados. Possivelmente, isso tenha ocorrido neste trabalho, pois três pontos mais à direita da reta foram originados do mesmo animal. Neste caso, independentemente da variação individual, PC apresenta sempre maiores valores de CLA cis-9 trans-11 $\mathrm{C}_{18: 2}$ que as demais fontes de carboidratos.

Assim, o ambiente ruminal proporcionado pelas dietas com polpa cítrica foi propício ao acúmulo de trans-11 $\mathrm{C}_{18: 1}$ como principal intermediário da biohidrogenação, e ações dietéticas ou de manejo que aumentem a concentração deste ácido graxo aumentarão o substrato para a síntese endógena do CLA. Por outro lado, a substituição do milho por polpa cítrica pode evitar a formação de trans- $10 \mathrm{C}_{18: 1}$ e de CLA trans- 10 cis-12 $\mathrm{C}_{18: 2}$ $\mathrm{e}$, assim, manter o teor de gordura do leite. Estas afirmações são válidas para as vacas de média produção utilizadas neste trabalho. O mesmo pode não ocorrer em animais de alta produção de leite, em decorrência do maior consumo e, conseqüentemente, da maior taxa de passagem da digesta, necessitando-se de mais pesquisas sobre o assunto.

\section{Conclusões}

A utilização de milho, polpa cítrica ou farelo de trigo no concentrado promove poucas alterações no perfil de ácidos graxos do leite. Entretanto, os resultados indicam que a polpa cítrica mantém a rota de biohidrogenação favorável ao acúmulo de trans-11 $\mathrm{C}_{18: 1}$ como o principal ácido graxo trans $-\mathrm{C}_{18: 1}$ no rúmen, aumentando o fornecimento de substrato para a síntese endógena de CLA. 
A adição de óleo de soja na dieta permite alterar o perfil da gordura do leite. A elevação da concentração de insaturados e do conteúdo de CLA e o aumento da participação de ácidos graxos de cadeia longa em detrimento dos ácidos graxos saturados oriundos da síntese de novo melhoram a qualidade nutricional do leite.

\section{Literatura Citada}

BATEMAN, H.G.; JENKINS, T.C. Influence of soybean oil in high fiber diets fed to nonlactating cows on ruminal unsaturated fatty acids and nutrient digestibility. Journal of Dairy Science, v. 81, p.2451-2458, 1998.

BAUMAN, D.E.; GRIINARI, J.M. Regulation and nutritional manipulation of milk fat: low-fat milk syndrome. Livestock Production Science, v.70, p.15-29, 2001.

BAUMAN, D.E.; BAUMGARD, L.H.; CORL, B.A. et al. [2000]. Biosynthesis of conjugated linoleic acid in ruminants. Disponível em: http://www.asas.org/ jas/symposia/proceedings/ 0937.pdf. Acesso em: 20/01/00.

BAUMGARD, L.H.; MATITASHVILI, E.; CORL, B.A. et al. Trans-10, cis-12 Conjugated linoleic acid decreases lipogenic rates and expression of genes involved in milk lipid synthesis in dairy cows. Journal of Dairy Science, v.85, p.2155-2163, 2002.

BRAFORD, B.J.; ALLEN, M.S. Milk fat responses to a change in diet fermentability vary by production level in dairy cattle. Journal of Dairy Science, v.87, p.3800-3807, 2004.

BESSA, R.J.B.; SANTOS-SILVA, J.; RIBEIRO, J.M.R. et al. Reticulorumen biohydrogenation and the enrichment of ruminant edible products with linoleic acid conjugated isomers. Livestock Production Science, v.63, p.201-211, 2000.

CHILLIARD, Y.; FERLAY, A.; MANSBRIDGE, R.M. et al. Ruminant milk fat plasticity: nutritional control of saturated, polyunsaturated, trans and conjugated fatty acids. Annales de Zootechnie, v.49, p.181-205, 2000.

CHOUINARD, P.Y.; GIRARD, V.; BRISSON, G.J. Fatty acids profile and physical properties of milk fat from cows fed calcium salts of fatty acids with varying unsaturation. Journal of Dairy Science, v.81, p.471-481, 1998.

CHRISTIE, W.W. A simple procedure for rapid transmethylation of glycerolipids and cholesterol esters. Journal of Lipid Research, v.23, p.1072, 1982.

DHIMAN, T.R.; SATTER, L.D.; PARIZA, M.W. et al. Conjugated Linoleic Acid (CLA) content of milk from cows offered diets rich in linoleic and linolenic acid. Journal of Dairy Science, v.83, p.1016-1027, 2000.

DRACKLEY, J.K.; BEAULIEU, A.D.; ELLIOTT, J.P. Responses of milk fat composition to dietary fat or nonstructural carbohydrates in Holstein and Jersey cows. Journal of Dairy Science, v.84, p.1231-1237, 2001.

EIFERT, E.C.; LANA, R.P.; LEÃO, M.I. et al. Efeito da combinação de óleo de soja e de monensina na dieta sobre o consumo e digestão em vacas lactantes. Revista Brasileira de Zootecnia, v.34, n.1, p.297-308, 2005.

EIFERT, E.C.; LANA, R.P.; LANNA, D.P.D. et al. Perfil de ácidos graxos do leite de vacas alimentadas com óleo de soja e monensina no início da lactação Revista Brasileira de Zootecnia, v.35, n.1, p.219-228, 2006a.

EIFERT, E.C.; LANA, R.P.; LANNA, D.P.D. et al. Consumo, produção e composição do leite de vacas alimentadas com óleo de soja e diferentes fontes de carboidratos na dieta Revista Brasileira de Zootecnia, v.35, n.1, p.211-218, 2006b.

ELLIOTT, J.P.; DRACKLEY, J.K.; FAHEY JR., G.C. et al. Utilization of supplemental fat by dairy cows fed diets differing in content of nonstructural carbohydrates. Journal of Dairy Science, v.78, p.1512-1525, 1995.
FOX, P.F.; McSWEENEY, P.L.H. Dairy chemistry and biochemistry. Great Britain: Blackie Academic \& Professional, 1998. p.67-145.

GRIINARI, J.M.; DWYER, D.A.; MCGUIRE, M.A. et al. Transoctadecenoic acids and milk fat depression in lactating dairy cows. Journal of Dairy Science, v.81, p.1251-1261, 1998.

GRUMMER, R.R. Effect of feed on the composition of milk fat. Journal of Dairy Science, v.74, p.3244-3257, 1991.

HALL, M.B. Recentes avanços em carboidratos não-fibrosos na alimentação de vacas leiteiras. In: SIMPÓSIO INTERNACIONAL EM BOVINOCULTURA DE LEITE: NOVOS CONCEITOS EM NUTRIÇÃO, 2., 2001, Lavras. Anais... Lavras: Universidade Federal de Lavras, 2001. p.149-159.

HARFOOT, C.G.; HAZLEWOOD, G.P. Lipid metabolism in the rumen. In: HOBSON, P.N.; STEWART, C.S. (Eds.) The rumen microbial ecosystem. Great Britain: Blackie Academic \& Professional, 1997. p.467-491.

JENKINS, T.C. Symposium: Advances in ruminant lipid metabolism - Lipid metabolism in the rumen. Journal of Dairy Science, v.76, p.3851-3863, 1993.

KALSCHEUR, K.F.; TETER, B.B.; PIPEROVA, L.S. et al. Effect of fat source on duodenal flow of trans- $\mathrm{C}_{18: 1}$ and milk fat production in dairy cows. Journal of Dairy Science, v.80, p.2115-2126, 1997.

KIM, Y.J.; LIU, R.H.; RYCHLIK, J.L. et al. The enrichment of a ruminal bacterium (Megasphaera elsdenii YJ-4) that produces the trans-10, cis-12 isomer of conjugated linoleic acid. Journal of Applied Microbiology, v.92, p.976-982, 2002.

LEIVA, E.; HALL, M.B.; Van HORN, H.H. Performance of dairy cattle fed citrus pulp or corn products as source of neutral detergent-soluble carbohydrates. Journal of Dairy Science, v.83, p.2866-2875, 2000.

LAWSON, R.E.; MOSS, A.R.; GIVENS, D.I. The role of dairy products in supplying conjugated linoleic acids to man's diet: a review. Nutrition Research Reviews, v.14, p.153-172, 2001.

LOOR, J.J.; HERBEIN, J.H. Reduced fatty acids synthesis and desaturation due to exogenous trans-10, cis-12-CLA in cows fed oleic or linoleic oil. Journal of Dairy Science, v.86, p.1354-1369, 2003.

MANSBRIDGE, R.J.; BLAKE, J.S. Nutritional factors affecting the fatty acid composition of bovine milk. British Journal of Nutrition, v.78, p.S37-S47, 1997 (supp1. 1).

NATIONAL RESEARCH COUNCIL - NRC. Nutrient requirements of dairy cattle. 7.ed. Washington, D.C.: National Academic Press, 2001. 381p.

NOUROOZ-ZADEH, J.; APPELQVIST, L.A. Cholesterol oxides in Swedish foods and food ingredients: milk powder products. Journal of Food Science, v.53, p.74-87, 1988.

PALMQUIST, D.L.; BEAULIEU, A.D.; BARBANO, D.M. Feed and animal factors influencing milk fat composition. Journal of Dairy Science, v.76, p.1753-1771, 1993.

PARODI, P.W. Conjugated linoleic acid and other anticarcinogenic agents of bovine milk fat. Journal of Dairy Science, v.82, p.1339-1349, 1999

PIPEROVA, L.S.; TETER, B.B.; BRUCKENTAL, I. et al. Mammary lipogenic enzyme activity, trans fatty acids and conjugated linoleic acids are altered in lactating dairy cows fed a milk fat depressing diet. Journal of Nutrition, v.130, p.2568-2574, 2000.

STATISTICAL ANALYSIS SYSTEM - SAS. SAS/STAT. User's guide. Version 6.12 4.ed. v.1. Cary: 1999. 890p.

SOLOMON, R.; CHASE, L.E.; BEN-GHEDALIA D. et al. The effect of nonstructural carbohydrate and addition of full fat extruded soybeans on the concentration of conjugated linoleic acid in the milk fat of dairy cows. Journal of Dairy Science, v.83, p.1322-1329, 2000 .

Recebido: 23/06/05 Aprovado: $15 / 03 / 06$ 